\title{
Still irresistible
}

\section{Half a century on, the Kondo effect continues to inspire.}

1964, in many respects, was a defining year for Japan. In October that year, the Olympic games were held in Tokyo. Less than a fortnight before the Olympic flame was lit, the first Shinkansen high-speed train entered service between Tokyo and Osaka - a new symbol of modernity and of rapid development towards a bright future. Evidently, Japan had stepped out of the long shadow cast over the country by the Second World War. People born in this period would in later decades be known as shinjinrui - 'the new breed'.

In the same year, a seed of a very different sort was planted: working in Tokyo's Electrotechnical Laboratory, physicist Jun Kondo came up with a solution ${ }^{1}$ to a long-standing problem in solid-state physics. He developed a "simple and universal model"2 that successfully explains why, in some dilute magnetic alloys, the electrical resistance does not decrease monotonically on cooling but rather starts increasing again below some characteristic temperature.

The phenomenon was first recorded in 1934 by Wander de Haas and colleagues at the Kamerlingh Onnes Laboratory in Leiden, The Netherlands. They studied the resistance of gold, copper and lead at low temperatures. The behaviour of gold was, however, troubling. "The resistance curve of the gold wires measured (not very pure) has a minimum", they reported ${ }^{3}$. That was unexpected - if resistivity stems from the scattering of electrons by lattice vibrations and imperfections, then it should keep decreasing with decreasing temperature. This phenomenological relation, known as Matthiessen's rule, didn't hold in gold, and similar behaviour was later found in other metals. From the outset, impurities were the prime suspect for the additional scattering and by the 1960s it had been established that the effect was due to the presence of magnetic impurities in normal metals.

But what was the mechanism that prevented electrons in these systems from gaining ever greater mobility? Kondo tackled the problem by starting from the so-called $s-d$ model, which describes the exchange scattering between the spin of an electron in a metallic $s$-band and the empty $d$-level of a magnetic impurity. Applying secondorder perturbation theory, he obtained a contribution to the alloy's resistivity that is proportional to the concentration of the impurities and logarithmically proportional to temperature. For the contribution to be increasing with decreasing temperature, and hence to explain the measurements made in Leiden and elsewhere, the $s-d$ interaction has to be antiferromagnetic - opposite alignments of the magnetic moments are favoured.

Kondo's solution was a great achievement, but it created a new problem. Owing to the logarithmic term, the resistivity becomes infinite at zero temperature - an unphysical implication. Many theorists were drawn to the 'Kondo problem', and it was quickly found that including even higher-order corrections did not help. But the exercise did lead to a definition of the so-called Kondo temperature, below which Kondo's perturbation approach broke down.

Gradually, there emerged a likely way-out of the problem: as the temperature goes down, the impurity spin is screened more and more by the conduction electrons. This scenario was confirmed in the early 1970s by Kenneth Wilson as a 'side result' of his numerical renormalization group ${ }^{4}$, a powerful technique that led not only to a complete solution of the Kondo problem but to a whole new way of approaching many-body problems - and to the 1982 Nobel Prize in Physics.

Further important theoretical results concerned the relation between Kondo's $s-d$ Hamiltonian and a Hamiltonian that had been introduced ${ }^{5}$ by Philip Anderson in 1961 that described a magnetic impurity with only one electron level. Both models give rise to a many-body effect called the Kondo resonance - a peak in the density of states at the Fermi level. Experimental observations of an increase in resistivity at low temperature were, in fact, the first signatures of a Kondo resonance.

Until the late 1990s, the Kondo effect had really only been exploited as a playground for theoretical solid-state physicists. But that changed in 1998, with advances in two branches of experimental physics: scanning tunnelling microscopy and quantum dots.

The scanning tunnelling microscope was originally invented to image surfaces with atomic resolution, but it was later shown to be capable of physically moving atoms as well. It proved to be the ideal tool for studying the Kondo physics of magnetic atoms on metallic surfaces ${ }^{6,7}$, leading to the observation ${ }^{8}$ of stunning 'quantum mirages' made by a team led by Donald Eigler at IBM.
Probably the best experimental platform for studying Kondo physics, however, is the quantum dot - a semiconductor heterostructure crafted in such a way that electrons are confined to a small spatial region within it. A spin can be associated with the dot and, importantly, that spin can be controlled; when the spin has the value one-half, the original Kondo scenario is recovered ${ }^{9}$. The possibility of preparing quantum dots with other spin values and of tuning a dot's electronic spectrum by applying a magnetic field has extended the scope of the Kondo effect well beyond its original context.

Kondo's seminal paper triggered an enormous amount of research and new physics, a process that continues today. A topic of ongoing interest is the 'Kondo cloud', the collective of electrons that screen the magnetic impurity and give rise to the Kondo resonance below the Kondo temperature experimental observations are eagerly awaited. Meanwhile, a new class of materials dubbed 'Kondo topological insulators' have been predicted ${ }^{10}$. And recently, the role of Kondo physics in a long-standing puzzle related to quantum point contacts (the ' 0.7 anomaly') has been revisited ${ }^{11}$.

1964 was a fruitful year for theoretical physics, and not only due to Kondo's contribution: John Bell conceived a framework for experimentally testing quantum non-locality; Murray Gell-Mann and George Zweig independently proposed the existence of quarks; and Robert Brout, François Englert and Peter Higgs... well, you probably know what they did. These are but a handful of examples of exceptional ideas that helped to develop a new breed of physicist in a post-war era that wasn't free of polarization, but characterized nevertheless by international exchange and collaboration.

\footnotetext{
References

1. Kondo, J. Prog. Theor. Phys. 32, 37-49 (1964).

2. Kondo, J. J. Phys. Soc. Jpn. 74, 1-3 (2004).

3. de Haas, W. J., de Boer, J. \& van den Berg, G. J. Physica 1, 1115-1124 (1934).

4. Wilson, K. G. Rev. Mod. Phys. 47, 773-840 (1975).

5. Anderson, P. W. Phys. Rev. 124, 41-53 (1961).

6. Madhavan, V., Chen, W., Jamneala, T., Crommie, M. F. \& Wingreen, N. S. Science 280, 567-569 (1998).

7. Li, J., Schneider, W.-D., Berndt, R. \& Delley, B. Phys. Rev. Lett. 80, 2893-2896 (1998).

8. Manoharan, H. C., Lutz, C. P. \& Eigler, D. M. Nature 403, 512-515 (2000).

9. Goldhaber-Gordon, D. et al. Nature 391, 156-159 (1998).

10. Dzero, M., Sun, K., Galitski, V. \& Coleman, P. Phys. Rev. Lett. 104, 106408 (2010).

11. Micolich, A. Nature Phys. 9, 530-531 (2013).
} 